\title{
High Power Narrow-Linewidth Fiber Lasers
}

\author{
Pu Zhou ${ }^{*}$, Wenchang Lai, Wei Liu, Can Li, \\ Rongtao Su, Hu Xiao, Jinyong Leng, Pengfei Ma** \\ College of Advanced Interdisciplinary Studies, National University of Defense Technology, \\ Changsha 410073, China \\ *E-mail: zhoupu203@163.com \\ **-mail: shandapengfei@126.com
}

DOI: 10.31868/RFL2020.40-41

High power narrow-linewidth fiber lasers have been under intensive investigation due to their potential applications in beam combination (coherent and spectral), gravitational wave detection (GVD), nonlinear frequency conversion and so on. In this talk, we will present the progress of high power narrow-linewidth fiber lasers in our lab [1-9] in the past few years and the very recent result.

Single frequency fiber laser is a special kind of narrow-linewidth fiber lasers with unique property in coherence length. One of the main scheme for power scaling of single-frequency fiber laser is to employ master oscillator power amplifier (MOPA) scheme. In the earlier stage, power scaling is mainly limited by the Stimulated Brillouin Scattering (SBS) effect, where up to 300 Watt level has been demonstrated by using large-mode-area fiber and therein shorter fiber length [1], the threshold of SBS can also be increased by introducing strain gradient and thermal gradient in the system [2]. Because of the newly discovered transverse modal instability (TMI) effect, further power scaling is limited by both SBS and TMI effects. Recently, by using polarizationmaintaining tapered Yb-doped fiber (T-YDF) and shorter seed wavelength, SBS and TMI can be well suppressed simultaneously, a $550 \mathrm{~W}$ single frequency fiber MOPA has been achieved with a slope efficiency of $80 \%$ [3].

The typical spectral linewidth for narrow-linewidth fiber laser is usually narrower than $0.3 \mathrm{~nm}$ (about $100 \mathrm{GHz}$ for $1 \mu \mathrm{m}$ laser). The output power can be significantly increased because much highly SBS threshold compared with single frequency fiber laser. There are several key issues for narrow-linewidth fiber lasers in addition to power scaling, that is, linewidth narrowing, TMI and even Stimulated Raman Scattering (SRS) effect suppressing. A kilowatt-level linearly-polarized fiber laser with a linewidth of about $1.8 \mathrm{GHz}$ has been achieved [4]. The power can be increased to 2 kilowatt-level by broadening the linewidth [5]. By using tandem-pumping scheme, which will result in less heat generation and thus higher MI threshold, near 4 kilowatt-level output power has been achieved [6], but the SRS effect should be considered since much longer active fiber is required for efficient pump absorption in tandem pumped ones [7].

From the other aspect of review, Raman gain can be used for power scaling single frequency or narrow linewidth fiber amplifier with special wavelength. For example, over $1 \mathrm{~kW}$ narrow-linewidth $1120 \mathrm{~nm}$ fiber laser was obtained with slope efficiency of $\sim 77 \%$ and near-diffraction-limited beam quality has been reported [8]. Single frequency fiber laser with $1120 \mathrm{~nm}$ wavelength was also achieved with a Raman fiber amplifier [9].

Recently, we have successfully demonstrated a diode-pumped narrow linewidth fiber amplifier. White noise modulation is employed to suppress the SBS, high-loss for HOM inside the fiber is employed to suppress the TMI, and up to $4.09 \mathrm{~kW}$ output power has been achieved. The output spectrum and beam quality measured at full power operation is shown in Figure 1. The SRS effect is suppressed by a factor of near $50 \mathrm{~dB}$, and the linewidth is about $0.28 \mathrm{~nm}$. The beam quality factor $\mathrm{M}^{2}$ is about 1.05 . To the 
best of our knowledge, this is the highest output power from a narrow linewidth fiber amplifier.
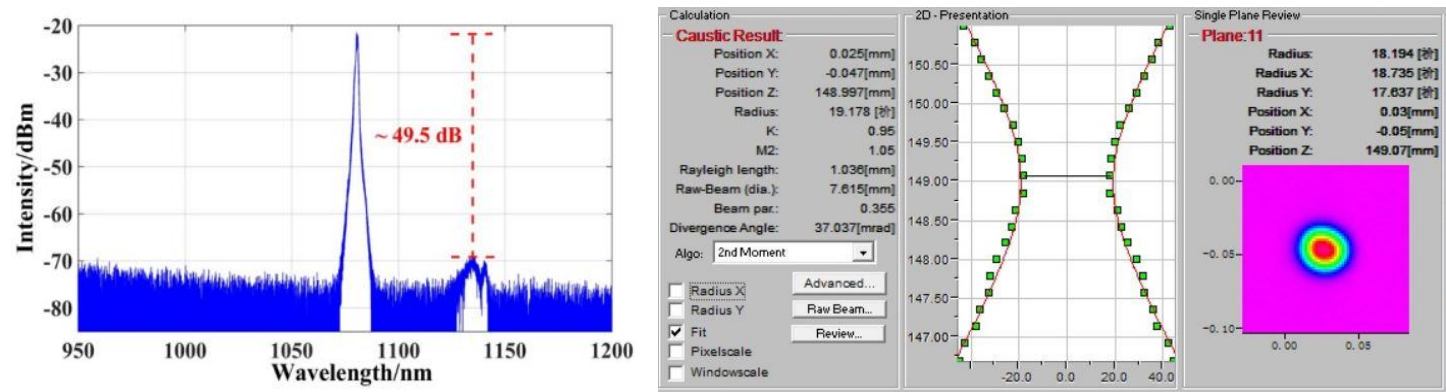

Fig. 1. Spectrum and beam quality measurement result at full power operation.

In the futher work, further narrowing the linewidth with new technique [10], increasing the output power by optimazing pump scheme [11] and expanding the operating wavelength [12] will be investigated in detail.

\section{Acknowledgment}

This work reviews in this paper is sponsored National Natural Science Foundation of China (NSFC) (No. 61705264), Hunan Provincial Innovation Construct Project (No. 2019RS3017), Innovative Research Team in Natural Science Foundation of Hunan province, China (No. 2019JJ10005) and Research Plan in Key Areas of Guangdong province, China (No.2018B090904001). The authors would like to express sincere thanks to Long Huang, Yu Miao, Daren Meng, Jiaxin Song, Rumao Tao, Yakun Liu, Hanwei Zhang, Man Jiang, Jian Wu, Yanxing Ma, et al. for their contribution to the work that support this talk.

\section{References}

[1] P. Ma, P. Zhou, Y. Ma, et al. Appl.Opt. 52, 4854 (2013).

[2] L. Huang, H. Wu, R. Li, et al. Opt. Lett. 42, 1-4 (2017)

[3] W. Lai, P. Ma, W. Liu, et al. Opt.Express, 28, 20908-20919 (2020)

[4] D.Meng, W.Lai, X.He, et al. Laser Phys. 29, 035103 (2019)

[5] P.Ma, R.Tao, R.Su, et al. Opt.Express, 24, 4187-4195 (2016)

[6] P.Ma, H.Xiao, D.Meng, et al. High Power Laser Science and Engineering, 6, e57 (2018)

[7] P.Zhou, H.Xiao, J.Leng, et al. Journal of the Optical Society of America B, 34, A29-A36 (2017)

[8] Y.Miao, P.Ma, W.Liu, et al. IEEE Access 6, 71988-71993 (2018)

[9] P.Ma, Y.Miao, W.Liu, et al. Opt. Lett. 45, 1974-1977 (2020)

[10] W. Lai, P. Ma, W. Liu, et al. IEEE Access 8, 71988-71993 (2020)

[11] W. Liu, P. Ma, Y. Miao, et al. IEEE J. Sel. Top. Quantum Electron, 24, 3100408 (2018)

[12] Y.Liu, R.Su, P.Ma, et al. Appl.Opt. 56, 4213 (2017) 